\title{
Classification of Lung Regions using Morphometrics for Chest CT Scans
}

\author{
K. Meenakshi Sundaram \\ Associate Professor / IT \\ Dhanalakshmi Srinivasan College of Engineering, \\ Coimbatore
}

\author{
C.S.Ravichandran, Ph.D \\ Professor \& Head / EEE (PG) \\ Sri Ramakrishna Engineering College, \\ Coimbatore
}

\begin{abstract}
Medical diagnosis is extremely important but complicated task that should be performed accurately and efficiently. Disease diagnosis is one of the applications where data mining tools are proving successful results. Chronic obstructive pulmonary disease (also known as COPD) is a condition that makes breathing difficult. Chronic Obstructive Pulmonary Disease (COPD) is the fourth leading cause of death worldwide and the only chronic disease with increasing mortality rates. COPD is the name for a group of lung diseases including chronic bronchitis, emphysema and chronic obstructive airways disease. The primary cause of COPD is smoking. Progression of the disease is irreversible but can be stabilized or prevented by quitting. If COPD is detected earlier, the formation of lung cancer is prevented. In CT scan may provide additional information and also it provides more detailed images of parts of the body that cannot easily be seen on a standard chest radiograph. First the input image is pre-processed then the lung region is segmented from that image, segmented the cavity region in that lung region, extracted some features for training the classifier and used the Extreme Learning Machine (ELM) classifier to identify the COPD affected lung. The preprocessing is done by using the median Filtering to avoid the noise in the input image and to increase the image quality. After the lung and cavity segmentation, some parameters are chosen to train the classifier to identify whether an $\mathrm{x}$-ray image is a normal or affected. The ELM Classifier is then trained using the parameters chosen from the sample chest CT scan images to identify the normal lung and tuberculosis affected lung.
\end{abstract}

\section{Keywords}

Chronic Obstructive Pulmonary Disease (COPD), ELM classifier, Median Filtering, Medical Imaging, Local Gabor XOR pattern (LGXP).

\section{INTRODUCTION}

Medical imaging has developed into one of the most important fields within scientific imaging due to the rapid and continuing progress in computerized medical image visualization and advances in analysis methods and computer-aided diagnosis. Several research applications are selected to illustrate the advances in image analysis algorithms and visualization.

Medical diagnostic and imaging system are ubiquitous in modern health care facilities. The advantages of early detection of potential lesions and suspicious masses within the bodily tissue have been well established. Chronic Obstructive Pulmonary Disease (COPD) is a most important disease but under-recognized cause of morbidity and mortality worldwide (Pauwels and Rabe, 2004). The occurrence of COPD in the general population is predictable to be, $\sim 1 \%$ across all ages rising steeply to $>10 \%$ amongst those aged 40 yrs (NCHS, 2001). The occurrence climbs appreciably higher with age. The
30 year for the global increase in COPD is from 1990-2020 is surprising. COPD is projected to move from the sixth to the third most common cause of death worldwide, at the same time as rising from fourth to third in terms of morbidity within the same time-frame (Murray and Lopez, 1997).

\section{RELATED WORK}

Image classification involves analyzing the numerical properties of various image features and organizing the data into categories. Usually, two phases of classification algorithms are employed: training and testing Kekre et al (2013).

In the initial training phase, characteristic properties of typical image features are identified and based on these, a unique description of each classification category, i.e. training class, is created. The description of training classes is an extremely important component of the classification process.

The motivating criteria for constructing training classes are that they are:

1. Independent, i.e. a change in the description of one training class should not change the value of another,

2. Discriminatory, i.e. different image features should have significantly different descriptions, and

3. Reliable, i.e. all image features within a training group should share the common definitive descriptions of that group.

In the next phase i.e. testing, accuracy of the classifier is measured. The accuracy can be determined by applying the classifier to an independent training set of objects with known classifications. Knowledge of the accuracy is necessary both in the application of the classifier and also in comparison of different classifiers.

A convenient way of building a parametric description of this sort is via a feature vector, where ' $n$ ' is the number of attributes which describe each image feature and training class.

This representation allows us to consider each image feature as occupying a point, and each training class as occupying a subspace (i.e. a representative point surrounded by some spread, or deviation), within the n-dimensional classification space. Viewed as such, the classification problem is that of determining to which sub-space class each feature vector belongs.

Classification can be linear or non linear. Linear classifiers are usually the fastest classifiers. It classifies the data on the basis of a linear combination of the characteristics based on which the classification is done. The classes are divided by a linear separator in the feature space. If the feature space is two dimensional, the separator is a line, it three dimensional the separator is a plane and if ' $p$ ' dimensional then the linear 
separator is a (p-1) dimensional hyper plane. Non linear classification is required when the class boundaries cannot be approximated well with linear hyper planes. Example of non linear classifier is K Nearest Neighbor.

There are mainly two ways of classification, supervised and unsupervised Rajiv Kapoor (2012). The difference between them lies in the fact that how the data is classified.

\section{i) Supervised classification}

In supervised classification, there are predetermined classes. Statistical processes (i.e. based on an a priori knowledge of probability distribution functions) or distribution free processes can be used to extract class descriptors. These classes can be regarded as a previously decided finite set. After classification, certain segment of data will be labeled with these classes. The task of the algorithm is to search for patterns and construct mathematical models. These models are then used to find out the measure of variance in the data and classify it. The examples of supervised classification are decision tree induction, naive bayes classifier etc.

\section{ii) Unsupervised classification}

Unsupervised classification relies on clustering algorithms to automatically segment the training data into prototype classes. In this type of classification, the classes are not pre decided. The basic task of the classifier is to develop the classes or the labels automatically. The algorithm is not told how the data is to be grouped; it is something it has to arrive at by itself. This is a difficult decision to make. The classifier looks for similarities between data and then determines which of these can form a group and can be classified under one label. The classes are also called clusters. The example of unsupervised classification is $\mathrm{K}$ means classification. In K-means, the classifier is told in advance, the no of clusters have to be formed.

\section{PROPOSED TECHNIQUE FOR THE IDENTIFICATION OF CAVITY}

The block diagram of the proposed approach is shown in figure 1. In this figure some sample chest CT scan images are taken with COPD and without COPD. The sample images are then preprocessed and then send for segmentation process. There segmenting the lung and cavity regions. After the lung and cavity regions are segmented from the sample images, some parameters are chosen to train the classifier. First the preprocessing is done to find weather the COPD is affected or not. After the preprocessing process, need to segment the lung and the cavity region. After that the chosen parameters are given to the classifier, here the Fuzzy Rule based classifier is used. The FRB classifier then identify whether the input chest CT scan image is affected by COPD or not by comparing the parameters from the sample images and from the input image.

\section{a. Pre-processing}

The input image is subjected to the pre-processing steps to make the image suitable for further process. The pre-process is used to load the input image to the MATLAB environment and it will remove the noise present in the input image. Here the median filter is used as pre-processing technique. The image is passed through the median filter to lower the noise and to get a better image. The input image is a normal RGB image. The RGB image is converted into grey scale image because the RGB format is not supported in Matlab. Then the grey scale image contains noises such as white noise, salt and pepper noise etc. This can be removed by using Median filter from the extracted lung image.

\section{Median Filter}

The median filter is a nonlinear digital filtering technique, frequently used to remove the noise. Such noise reduction is a typical pre-processing step to improve edge detection on an image.

The sliding median filter of a pre-defined window size $W \times W$ centered at $i=\left(i_{1}, i_{2}\right)$ moves regularly over the noisy image, $g$, chooses the median, $\mu$ of the pixels within the spatial positions $\Omega_{i}^{W}$ around $i$ to have $g(i)$ replaced by $\mu$. For the set of pixels within a square window $W D \times W D$, centered at $i=\left(i_{1}, i_{2}\right)$ and defined spatially by the below equation, the median, $\mu$ of the pixels in $\Omega_{i}^{W}$ is

$$
u(i)=\mu(i)=\text { median }\left\{g(j) / j \epsilon \Omega_{i}^{W}\right.
$$

Thus the output of the median filter is that value $\theta$ which produces the least sum of absolute errors with all the pixels in the local neighborhood defined by the mask. The output of the median filter at spatial position $i$ can also be given as

$$
u(i)=\mu(i)=\arg _{\theta}^{\min } \sum_{r \in \Omega_{i}^{W}}|g(r)-\theta|
$$




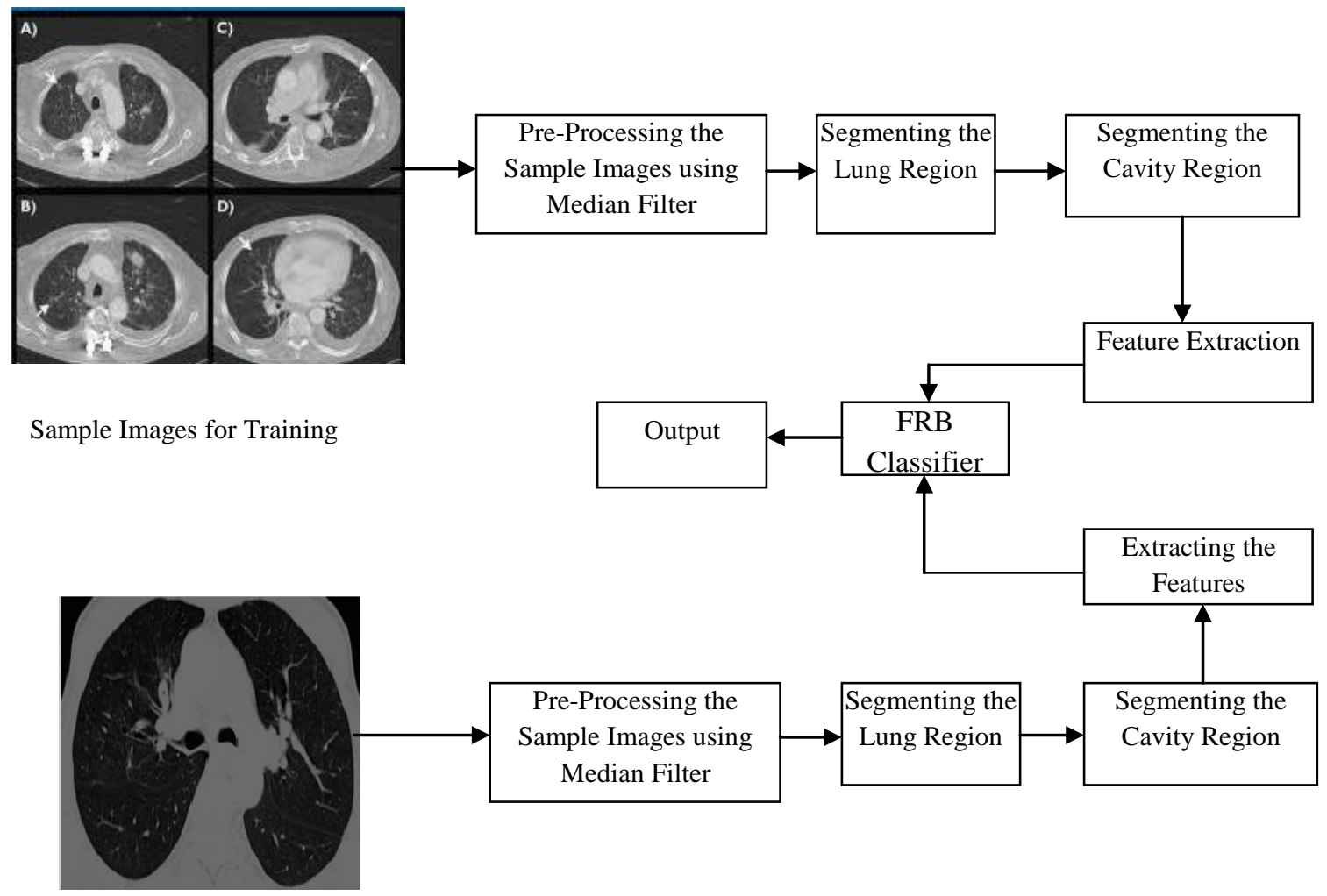

Input Image to Test

Figure 1: Block Diagram for Proposed Technique

\section{b. Lung Segmentation}

Lung segmentation is a process of segmenting the lungs from the chest CT scan image. The normal process of region growing technique for segmenting the lungs is shown in the figure 2 . First choose a pixel from the chest CT scan image as default. Then need to set a threshold value for comparison to find the pixel intensity for the lung area in the chest CT scan. The default pixel which chosen is compared with the adjacent pixel values. If the difference between the default pixel and the adjacent pixel is greater than the threshold value, have to exclude that adjacent pixel. If the difference between the default pixel and the adjacent pixel is less than the threshold value, have to include that adjacent pixel for region growing. Compare all the pixels except the left pixels with its adjacent pixels by keeping one pixel as default. The process of normal region growing technique is shown in the Figure 2.

In this paper, comparing the normal region growing technique with the Local Gabor XOR Pattern (LGXP) based region growing technique to segment the lungs from the chest CT scan image Shufu Xie et al (2010). The LGXP technique is used to find the texture image.

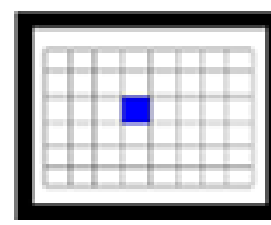

Choose a Pixels Default

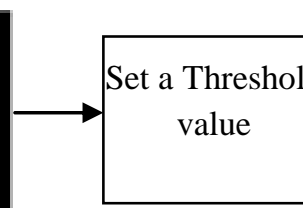

.

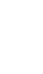

Figure 2: Block Diagram of normal Region Growing Technique 
The LGXP based region growing technique is as follows. In LGXP technique, apply the Gabor Phase Technique on every pixel in the chest CT scan image. The Gabor Phase Technique will convert all the pixel values to phase values (0 to 360). After converting all the pixel values to phase values, find these phase values comes under which quadrant. Each quadrant has certain values. For the first quadrant the value is zero and for the second quadrant the value is one and for the third quadrant the value is two and for the fourth quadrant the value is three. After that choose a default phase value of a pixel and check under which quadrant this phase value comes and assign respective quadrant value to that pixel.After assigning respective quadrant value to the default pixel, check the adjacent pixel's phase and values and assign the respective quadrant values to those adjacent pixels. Then convert the adjacent pixel's value as zero which has the same quadrant value of the default pixel.If the adjacent pixels value does not have the same quadrant value of the default pixel, convert the adjacent pixel's value as one. Now the pixel values would be like binary values as zeros and ones. After converting the pixel values as binary format, make that binary format as decimal value and apply that decimal value to the default pixel. The process of taking the binary value is shown in the figure. Likewise apply this LGXP process for all the pixels in the chest CT scan by keeping one pixel as default. The sample process of LGXP technique is shown in the Figure.3.

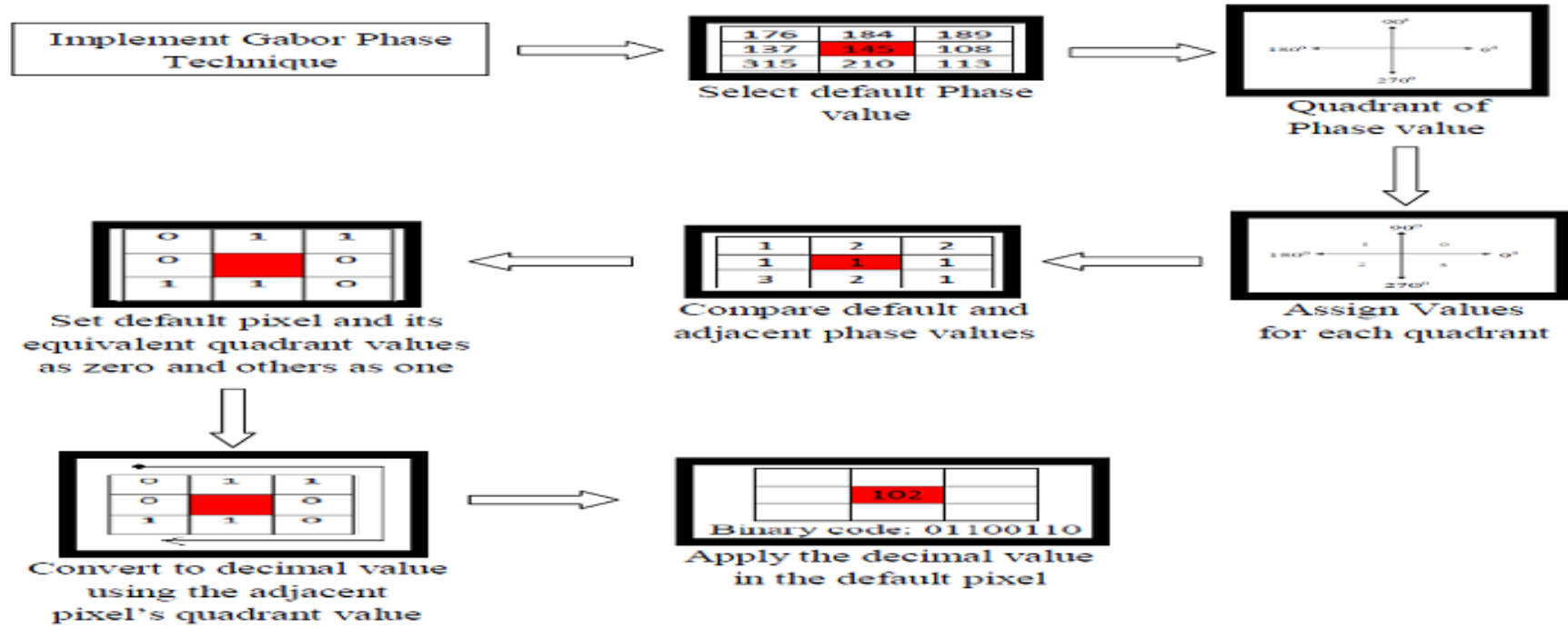

Figure 3: Block diagram of LGXP Technique

After applying the LGXP technique in all the pixels, then implement the region growing technique for segmenting the and LGXP based region growing technique. By comparing both the techniques check whether the same pixel as default. During this process, if the difference between the adjacent pixel and the default pixel got the value as less than the threshold value on both the techniques separately, include that adjacent pixel for region growing or else need to exclude that adjacent pixel. But the adjacent pixel and the default pixel which have chosen to compare should be same on both the techniques.

\section{c. Feature Extraction}

After finding the regions, extract some features to diagnose the disease in the lung. To discover the disease in the lung, have to feed the extracted feature into the classifier, because the extracted features will give vital information about the region which is used to train the classifier. In this paper an ELM classifier is used for feature extraction. The features need to extract are number of cavities in the lung region, minimum area of cavity region, maximum area of cavity region, total number of pixels in each cavity, maximum repeated pixel intensity in the cavity region and maximum repeated pixel in the lung region to find the total number of cavities in the lung region. Because the normal lung would also have some cavities present in its region. So to distinguish the normal lung image and the COPD affected lung should find the total numbers of cavities present in the lung region and give the result to the ELM classifier.

Extreme Learning Machine (ELM) meant for Single Hidden Layer Feed-Forward Neural Networks (SLFNs) will randomly lungs using the phase value of the pixels from the LGXP process. Then compare the normal region growing technique select the input weights and analytically determine the output weights [18]. This algorithm tends to afford the best generalization performance at extremely fast learning speed.The structure of ELM network is shown in figure 4. ELM contains an input layer, hidden layer and an output layer.

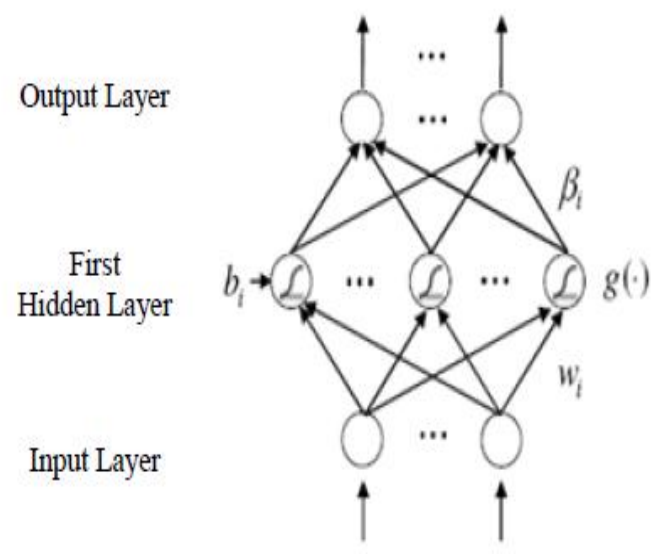

Figure 4: Structure of ELM

The ELM has several interesting and significant features different from traditional popular learning algorithms for feed forward neural networks. These include the following: 
- The learning speed of ELM is very quick when compared to other classifier. The learning process of ELM can be performed in seconds or less than seconds for several applications.

- The ELM will attain the results directly without any difficulties. The ELM learning algorithm is much simple than the other learning

Extreme Learning Machine Training Algorithm, it uses a finite number of input-output samples for training. If there are $\mathrm{N}$ samples are considered in the lung cavity region as $\left(X_{i}, t_{i}\right)$, where $X_{i}=\left[X_{i 1}, X_{i 2} \ldots \ldots X_{i n}\right]^{T} \in R^{n} \quad$ and $t_{i}=\left[t_{i 1}, t_{i 2} \ldots \ldots t_{i m}\right]^{T} \in R^{n}$ are the cavity and the area of the cavity region, then the standard SLFN with $\mathrm{N}$ hidden neurons and activation function $\mathrm{g}(\mathrm{x})$ is defined as:

$$
\sum_{i=1}^{\bar{N}} \beta_{i} g\left(w_{i} . X_{j}+b_{j}\right)=0_{j}, \quad j=1, \ldots \ldots . . N
$$

Where $w_{i}=\left[w_{i 1}, w_{i 2} \ldots \ldots w_{i n}\right]^{T}$ represents the weight vector that links the ith hidden neuron and the input neurons, $\beta_{i}=$ $\left[\beta_{i 1}, \beta_{i 2} \ldots \ldots \beta_{i n}\right]^{T}$ represents weight vector that links the ith neuron and the output neurons, and $b_{i}$ represents the threshold of the ith hidden neuron. The "." in $\mathrm{w}_{\mathrm{i}} . \mathrm{x}_{\mathrm{j}}$ indicates the inner product of $\mathrm{w}_{\mathrm{i}}$ and $\mathrm{x}_{\mathrm{j}}$. The SLFN try to reduce the difference between $\mathrm{o}_{\mathrm{j}}$ and $\mathrm{t}_{\mathrm{j}}$. More in a matrix format as $\mathrm{H} B=\mathrm{T}$, where $H\left(a_{1}, \ldots \ldots ., a_{\widetilde{N}}, b_{1}, \ldots \ldots \ldots b_{\widetilde{N}}, X_{1}, \ldots \ldots \ldots X_{\widetilde{N}}\right.$

$$
=\left[\begin{array}{ccc}
g\left(a_{1}, b_{1}, X_{1}\right) & \cdots & g\left(a_{\widetilde{N}}, b_{\widetilde{N}}, X_{1}\right) \\
\vdots & \ddots & \vdots \\
g\left(a_{1}, b_{1}, X_{N}\right) & \cdots & g\left(a_{\widetilde{N}}, b_{\widetilde{N}}, X_{N}\right)
\end{array}\right]_{N X \widetilde{N}}
$$

$\beta=\left[\begin{array}{c}\beta_{1}^{T} \\ \vdots \\ \beta_{\widetilde{N}}^{T}\end{array}\right]_{\widehat{N X m}}$ and $T=\left[\begin{array}{c}t_{1}^{T} \\ \vdots \\ t_{\widetilde{N}}^{T}\end{array}\right]_{N X m}$

The result reduces the norm of this least squares equation is:

$$
\tilde{\beta}=H^{+} T
$$

Where $H^{+}$the Moore-Penrose is generalized inverse Karpagachelvi et al (2011) of the hidden layer output matrix $H$. The ELM algorithm which consists of only three steps, can then be summarized as

Step 1: Given a training set

$\aleph=\left\{\left(X_{i}, t_{i}\right) \mid X_{i} \in R^{m}, i=1, \ldots \ldots, N\right\}$ activation function $\mathrm{g}(\mathrm{x})$, and hidden number node $\widetilde{N}$,

1) Assign random hidden nodes by randomly generating parameters $\left(a_{i}, b_{i}\right)$ according to any continuous sampling distribution, $i=1, \ldots ., \widetilde{N}$

2) Calculate the hidden layer output matrix $H$

3) Calculate the output weight $\beta: \tilde{\beta}=H^{+} T$

Then find the maximum repeated pixel intensity in the cavity regions of a lung. To discover the maximum repeated pixel, first have to find the intensities of all the pixels in each cavity of a lung by implementing histogram and thereafter need to compare all the pixels of every cavity with each other. After discovering the maximum repeated pixel in the cavities of a lung, have to give the result to the classifier. Similarly, find the maximum repeated pixel in the whole lung region and give the result to the classifier. The classifier detect the lung is affected by COPD or not by comparing all the features.

\section{TRAINING AND TESTING USING ELM CLASSIFIER}

Some of the data features are to be taken to identify the normal lung region and COPD affected lung by this the classifier is trained. The data features will then train the classifier and the classifier will find whether the given CT scan image is normal or abnormal. The data features which have chosen for training the ELM classifier are number of cavities in the lung region, maximum area of cavity in the lung region, minimum area of cavity in the lung region, total number of pixels in each cavity, maximum repeated pixel in the cavity regions together and maximum repeated pixel in the lung region. After computing all the data features, have to give the values to the classifier. For instance, choosing three normal CT scan images and three abnormal CT scan images, need to calculate all the six data features separately for all the CT scan images had chosen. After calculating all the six data features for every chosen CT scan images, have to give the result to the ELM classifier. Using those results train the classifier to identify the normal and abnormal lung from the given CT scan image. After the ELM classifier is trained, give a new CT scan image to find whether it has COPD or not. Thereafter, the six data features such as number of cavities in the lung region, maximum area of the cavity region, minimum area of the cavity region, total number of pixels in each cavity, maximum repeated pixel in the cavity region and maximum repeated pixel in the lung region are computed for the new CT scan image. The computed values of all the six data features are then give to the ELM classifier. The ELM classifier is then comparing the values of all the six data features with the stored values of normal and abnormal CT scan images. Because during training have stored all the six data features of the five normal CT scan images and five abnormal CT scan images. After comparison, the ELM classifier will identify whether the given CT scan image comes under normal category or abnormal category.

\section{RESULTS AND DISCUSSION}

The experimental result is conducted in MATLAB. The figure 5 shows the normal and abnormal lung images.



(a)Normal Image (b) Abnormal Image

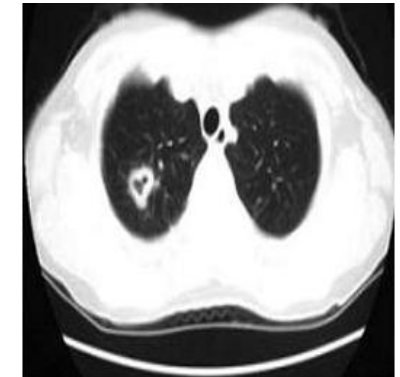

Figure 5: Sample images of normal and abnormal lungs images

The sample images are taken and the images are filtered using Laplacian Gaussian filter. The filtering technique is used to remove the noises and it improves the quality of the images as shown in the figure 6 .

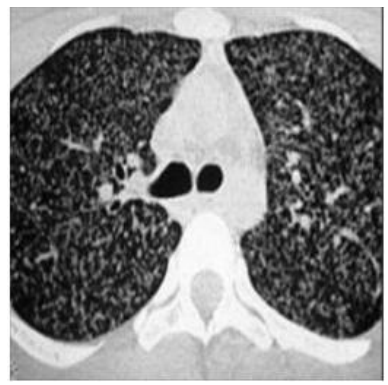
filtering process
(a)Normal Image after

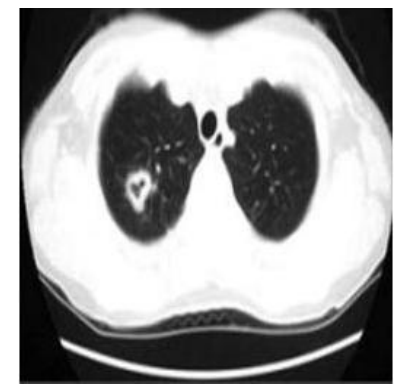

(b) Abnormal Image after filtering process
Figure 6: Sample image of Lungs after Filtering process

The sample images after applied the filtering technique are given to the process of lung segmentation. The lung segmentation process only segments the lung region from the 
sample CT scan images. The Figure 7 shows a sample image of segmented lungs with COPD and without COPD.

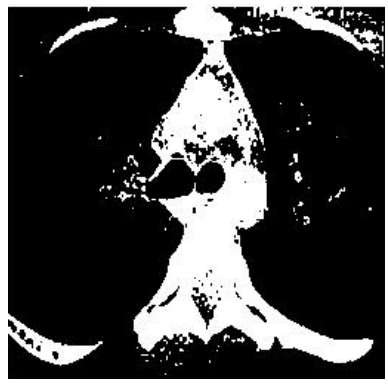

(a)Normal Image after segmentation process Figure 7: Sample image

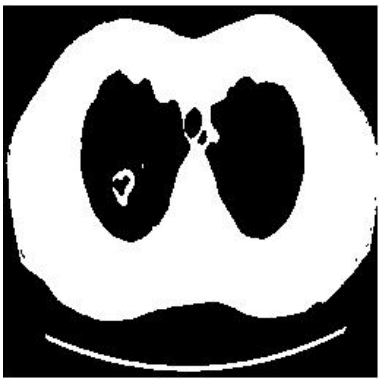

(b) Abnormal Image after segmentation process process

After the lung is segmented from the sample images, have to segment the cavities from the lung region. Using the cavities in the lung region identify whether a lung is COPD affected or not. The Figure 8 shows a sample image of segmented cavities and segmented cavities with CT scan image for the COPD affected lung.

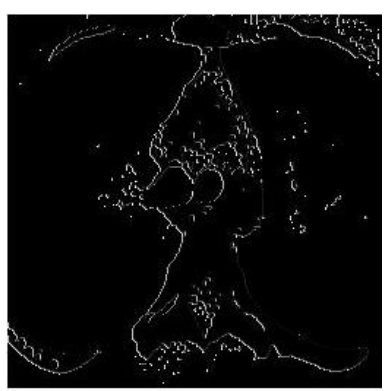

(a)Normal Image after segmenting cavities

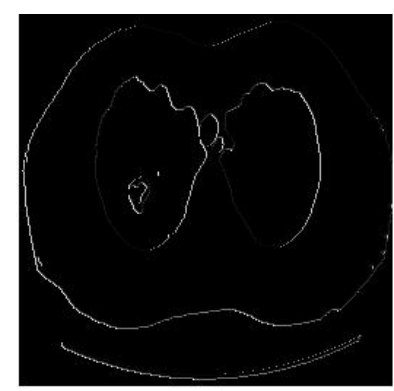

(b) Abnormal Image after segmenting cavities
Figure 8: Sample image of Lungs after segmenting the cavities

\subsection{Performance Analysis Using Evaluation Metrics}

The evaluation of the COPD identification of the images is carried out using the following metrics,

Where,

$$
\begin{gathered}
\text { Sensitivity }=T P /(T P+F N) \\
\text { Accuracy }=(T N+T P) /(T N+T P+F N+F P)
\end{gathered}
$$

True Positive TP, True Negative TN, False Negative FN, False Positive FP

Sensitivity is the proportion of true positives that are correctly identified by a diagnostic test. It shows how good the test is at detecting a disease.

Accuracy is the proportion of true results, either true positive or true negative, in a population. It measures the degree of veracity of a diagnostic test on a condition.

Table 1 shows the accuracy comparison between proposed technique and the existing technique. The tabular column shows that the proposed technique gives better performance than the existing technique.
Table: 1 Comparative analysis of existing technique with our proposed technique

\begin{tabular}{|c|c|c|c|c|c|c|}
\hline Techniques & TP & TN & FP & FN & Sensitivity & Accuracy \\
\hline $\begin{array}{c}\text { Existing } \\
\text { SVM } \\
\text { Technique }\end{array}$ & 3 & 9 & 1 & 1 & 0.9 & 0.857 \\
\hline $\begin{array}{c}\text { Proposed } \\
\text { Technique }\end{array}$ & 2 & 7 & 1 & 0 & 0.75 & 0.897 \\
\hline
\end{tabular}

\section{CONCLUSION}

In this paper, proposed an efficient technique for the detection of COPD in the lungs using CT scan Images. The proposed technique contains pre-processing, lung segmentation, cavity segmentation, feature extraction, training and testing using ELM classifier. The ELM classifier is efficient and simple in nature. The performance of the proposed technique and the existing technique is analyzed using evaluation metrics. To evaluate these metrics, should need some terms like True Positive, True Negative, False Positive and False Negative. After evaluating these metrics it shows that the performance of proposed technique is better when compared to the existing technique in terms of accuracy. The result shows that the accuracy of proposed technique is better than the existing techniques.

\section{REFERENCES}

[1] Abdel-Motaleb, Ibrahim, "Artificial intelligence algorithm for heart disease diagnosis using Phonocardiogram signals", IEEE, 2012.

[2] Barnes PJ. Future advances in COPD therapy. Respiration 2001; 68: 441-448.

[3] Farzaneh Keyvanfard "Feature selection and classification of breast MRI image" Artificial Intelligence and Signal Processing AISP 2011 International Symposium on (2011) pp. $54-58$.

[4] Hegedus, L. (2004) Thyroid ultrasound as a screening tool for thyroid disease. Thyroid, vol. 14, no 11 (2004), pp. $879-880$.

[5] Ming-Feng Wu, Chao-Ling Chen ; Chih-Yu Wen ; JengYuan Hsu, "Design of Pervasive Rehabilitation Monitoring for Chronic Obstructive Pulmonary Disease”, IEEE, 2013.

[6] Murray CJ, Lopez AD. Alternative projections of mortality and disability by cause 1990-2020: Global Burden of Disease Study. Lancet 1997; 349: 1498-1504.

[7] Murray CJ, Lopez AD, Mathers CD, Stein C. The Global Burden of Disease 2000 Project: aims, methods, and data sources. Global Programme on Evidence for Health Policy Discussion, paper No. 36. Geneva, World Health Organization, 2001.

[8] National Center for Health Statistics. Vital statistics of the United States, 1950-1999, Vol. 2. Mortality, parts A and B. Washington DC, Public Health Service, 2001.

[9] Pauwels RA, Rabe KF. Burden and clinical features of chronic obstructive pulmonary disease (COPD). Lancet 2004; 364: 613-620.

[10] Rajkot, "Feature Extraction of Bone Scintigraphy for Diagnosis of Diseases", International Conference on Communication Systems and Network Technologies, 2012.

[11] B. Magesh, P. Vijayalakshmi, M. Abirami, "Computer Aided Diagnosis System for the Identification and 
Classification of Lessions in Lungs", International Journal of Computer Trends and Technology- May to June Issue 2011.

[12] V.M. Katoch, "Newer diagnostic techniques for tuberculosis", Central JALMA Institute for Leprosy \& Other Mycobacterial Diseases (ICMR), Agra, India Received July 16, 2003.

[13] Giger, M.L., N. Karssemeijer, and S.G. Armato, Guest editorial computer- aided diagnosis in medical imaging, pp. 1205-1208.

[14] Y.Uchiyama, S.Katsuragawa, H.Abe, J.Shiraishi, F.Li, Q.Li, C.-T.Zhang, K.Suzuki and K.Doi. Quantitive computerised analysis of diffuse lung disease in highresolution computed tomography, Med. Phys. 30 (9) September 2003, pp. 2440-2453
[15] Sluimer IC, van Waes PF, Viergever MA, van Ginneken B. Computer-aided diagnosis in high resolution CT of the lungs, Med Phys. 30 (12) December 2003, pp. 3081-90.

[16] N. Lee et al., "Fatty and fibroglandular tissue volumes in the breasts of women 20-83 years old: Comparison of Xray mammography and computer-assisted MR imaging," Amer. J. Roentgenol ., vol. 168, pp. 501-506, 1997.

[17] Shufu Xie, Shiguang Shan, Xilin Chen, Jie Chen, "Fusing Local Patterns of Gabor Magnitude and Phase for Face Recognition", IEEE Transactions on Image Processing, Vol. 19, No. 5, PP. 1349- 1361, 2010.

[18] S. Karpagachelvi, Dr. M. Arthanari and M.Sivakumar," Classification of Electrocardiogram Signals With Extreme Learning Machine and Relevance Vector Machine", IJCSI International Journal of Computer Science Issues, Vol. 8, Issue 1, January 2011. 